\title{
Side chain length is more important than stereochemistry in the antibacterial activity of enantiomerically pure 4-aminoalcohol quinoline derivatives
}

\author{
Alexia Jonet, Alexandra Dassonville-Klimpt, Pascal Sonnet and Catherine Mullié \\ The Journal of Antibiotics (2013) 66, 683-686; doi:10.1038/ja.2013.71; published online 3 July 2013
}

Keywords: antibacterial activity; 4-aminoalcohol quinoline; mefloquine; stereochemistry

Mefloquine (MQ) is a quinoline methanol derivative used in the preventive and curative treatment of malaria. MQ was also previously found to be effective on Gram-positive bacteria such as Staphylococcus aureus, Streptococcus pneumoniae or, to a lesser extent, Enterococcus faecalis $^{1}$ as well as on Mycobacterium avium complex. ${ }^{2}$ In addition, it has been reported as a potential inhibitor of efflux pumps in Gramnegative bacteria. ${ }^{3}$ However, the use of $\mathrm{MQ}$ as an antibiotic has been limited by relatively high minimum inhibitory concentrations (MICs) as well as adverse effects, such as neurotoxicity, ${ }^{4}$ highlighted through its use as an antimalarial drug. A way to enhance the activity and reduce the neurotoxicity of MQ derivatives could be the synthesis of enantiomerically pure 4-aminoalcohol quinolines, as enantiomerism could have a part in the in vitro activity and toxicity of these molecules. MQ possesses two asymmetric carbon atoms and is commercially available as a racemic mixture of its erythroenantiomers (Figure 1). Inhibitory concentrations $50\left(\mathrm{IC}_{50}\right)$ values for the $(+)$-enantiomer of MQ were found to be lower than those of the $(-)$-enantiomer by a factor of 1.6-1.8 on some strains (D6 and W2), ${ }^{5}$ and we recently showed that $(S)$-4-aminoalcohol quinolines derivatives were 2- to 15-fold more active than their $(R)$ counterparts on W2 and 3D7 Plasmodium falciparum strains. ${ }^{6}$ The antibacterial activity of MQ has also recently been proposed to be dependent on enantiomerism, as $(+)-(11 S, 12 R)$ MQ was found to be more efficient in clearing $M$. avium from tissues of infected mice. ${ }^{7}$

Furthermore, the (-)-enantiomer was found to block central nervous system adenosine receptors, whereas the $(+)$-enantiomer did not. This blockage of central adenosine receptors by the (-)enantiomer is believed to result in neuropsychiatric symptoms associated with MQ. ${ }^{8}$ With these differences in mind, a new enantioselective pathway to synthesize pure enantiomers of MQ amino-analogs was recently proposed..$^{9}$ The structures of these derivatives are displayed in Figure 1. As they only bear one asymmetric carbon at the position 11, the importance of the stereochemistry of this asymmetric carbon and of the length of the lateral chain on the antibacterial activity have been evaluated in this work.

MQ and MQ derivatives were tested on Escherichia coli DSM 1103, Pseudomonas aeruginosa DSM 1117, S. aureus CIP 103.429 and E. faecalis CIP 103.214. In addition, $P$. aeruginosa AM85, a strain formerly identified as resistant to ciprofloxacin through an effluxmediated mechanism, ${ }^{10}$ as well as ten recent clinical strains (isolated in 2010) of S. aureus resistant to methicillin, ciprofloxacin and levofloxacin were tested for their susceptibility to MQ derivatives. For MIC determinations, the broth microdilution technique was carried out as advised by the Clinical and Laboratory Standards Institute using drug concentrations ranging from 0.0625 to $128 \mu \mathrm{g} \mathrm{ml}^{-1}$. $^{11}$ In the series of MQ derivatives with a linear side chain, with the exception of $(S)-\mathbf{6}$, compounds were found to be at least as effective as MQ on S. aureus and E. faecalis. For these species, the lower MICs were witnessed for compounds 3, 4, 5 and 7 with a 4- to 16-fold gain in activity compared with MQ and MICs ranging from 1 to $4 \mu \mathrm{g} \mathrm{ml}^{-1}$ (Table 1 ). In addition, in vitro susceptibility evaluation showed that $(R)$ - and $(S)$-enantiomers displayed similar MICs against the Gram-positive bacteria tested. The only noticeable exception was compound $(R)-6$ that was found to be more effective than (S)-6 (Table 1). For E. coli, MQ derivatives with shorter side chains (compounds 1, 2 and (S)-3) were the most effective as well as compound $\mathbf{8}$, bearing an hydroxyphenyl substituent (Table 1). A twofold difference in favor of the $(R)$-enantiomers was found for 1, 2 and 8. Interestingly, for methicillin-resistant $S$. aureus strains, $\mathrm{MIC}_{50}$ and $\mathrm{MIC}_{90}$ (the MIC capable of inhibiting the growth of at least 50 and $90 \%$ of the strains in the panel tested, respectively) for MQ and MQ derivatives only differed by a two-fold factor, whereas this factor was of eightfold for ciprofloxacin. The overall antibacterial activity of 
<smiles>O[C@H](c1cc(C(F)(F)F)nc2c(C(F)(F)F)cccc12)[C@@H]1CCCCN1</smiles>

(-)-(11R, 12S)-erythro-mefloquine<smiles>[R]NC[C@@H](O)c1cc(C(F)(F)F)nc2c(C(F)(F)F)cccc12</smiles>

(11R)-4-aminoalcohol quinoline derivatives<smiles>O[C@H](c1cc(C(F)(F)F)nc2c(C(F)(F)F)cccc12)[C@@H]1CCCCN1</smiles>

(+) -(11S, 12R)-erythro-mefloquine<smiles>[R]NC[C@@H](O)c1cc(C(F)(F)F)nc2c(C(F)(F)F)cccc12</smiles>

(11S)-4-aminoalcohol quinoline derivatives

\begin{tabular}{cc}
\hline R & Compound number \\
\hline Butyl & $\mathbf{1}$ \\
Pentyl & 2 \\
Hexyl & 3 \\
Heptyl & 4 \\
Octyl & 5 \\
Nonyl & 6 \\
Phenyl & 7 \\
Para-hydroxyphenyl & $\mathbf{8}$ \\
\hline
\end{tabular}

Figure 1 Structures of mefloquine $(\mathrm{MQ})$ and 4-aminoalcohol quinoline derivative enantiomers.

4-aminoalcohol quinoline derivatives on Gram-positive bacteria did not seem to be dependent on their stereochemistry but rather on the length of the side chain, as compounds bearing 6, 7 and 8 carbons on this side chain were the more efficient on both methicillin-sensitive and -resistant $S$. aureus strains as well as on E. faecalis. This finding is somewhat at odds with what was previously described for the antibacterial activity of MQ, as stereochemistry was recently reported to be of importance in MQ activity in a mouse model of $M$. avium infection, with the $(+)-(11 S, 12 R)$-enantiomer of MQ being the more efficient. ${ }^{7}$ In addition, the antimalarial activity of the same MQ derivatives, as those tested here, was shown to be significantly better for $(S)$-enantiomers on two P. falciparum strains, one resistant and one sensitive to chloroquine. ${ }^{6}$ The mechanism underlying the differential activity of MQ and MQ derivative enantiomers against $P$. falciparum has not clearly been elucidated but a possible explanation might reside in the stereospecific recognition by transporters located on the digestive vacuole membrane such as Plasmodium falciparum Chloroquine Resistance Transporter (PfCRT) or Plasmodium falciparum MultiDrug Resistance 1 (PfMDR1), which enable quinoline derivatives to reach and/or remain at their site of action. ${ }^{12}$ A hypothesis to explain the lack of stereospecificity on $S$. aureus and E. faecalis might, therefore, be that MQ derivatives do not depend on a transport system to enter Gram-positive bacteria, whereas such a system, possibly allowing the preferential entry of one of MQ or MQ derivatives enantiomers, could be necessary to enable their activity on $M$. avium and/or Gram-negative bacteria. Indeed, whereas the MQ derivatives tested in this work were inactive against $P$. aeruginosa, possibly through lack of penetration, an activity dependant on the stereochemistry at the position 11 was witnessed for E. coli, with $(R)$-enantiomers of MQ derivatives having shorter side chains being the more efficient (Table 1).

$\operatorname{cog} P$ values calculated for MQ derivatives ranged from 3.51 for 8 to 5.54 for 6 (Pallas 3.5 software, Compudrug International, Inc., Sedona, AZ, USA) and fell within the range of values usually found for drugs (that is, 2-5). ${ }^{13}$ Moreover, MICs witnessed for the more active compounds were of $1-2 \mu \mathrm{g} \mathrm{ml}^{-1}$ for Gram-positive bacteria and $8-16 \mu \mathrm{g} \mathrm{ml}^{-1}$ for E. coli. If a parallel is drawn with MQ pharmacokinetics, the active concentrations against Grampositive bacteria, but not those against $E$. coli, should be achievable in clinical situations. Indeed, previously published papers have shown that MQ concentrations can reach up to $3 \mu \mathrm{g} \mathrm{ml}^{-1}$ after a $25-\mathrm{mg} \mathrm{kg}^{-1}$ oral dose of MQ. ${ }^{14}$ Interestingly, the screening of 3, 4 and 5 against recent clinical strains of $S$. aureus resistant to both methicillin and fluoroquinolones showed that MQ derivatives retained their activity against those strains, hence offering an alternative to treat such resistant strains and pointing out that a different mechanism of action from that of $\beta$-lactams and fluoroquinolones could be involved. 


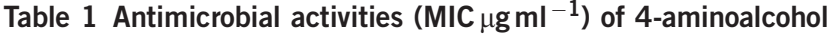
quinoline derivatives

\begin{tabular}{|c|c|c|c|c|c|c|}
\hline \multirow[b]{3}{*}{ Compound } & \multirow{2}{*}{$\begin{array}{c}\text { S. aureus } \\
\text { CIP }\end{array}$} & \multicolumn{4}{|c|}{ E. faecalis E. coli } & \multirow[b]{2}{*}{ aeruginosa } \\
\hline & & MRSA & CIP & $D S M$ & P. aeruginosa & \\
\hline & 103.429 & (10 strains) & 103.214 & 1103 & DSM 1117 & AM 85 \\
\hline Ciprofloxacin & 0.25 & $16 / 128^{a}$ & 0.25 & 0.0625 & 0.125 & 16 \\
\hline Mefloquine & 16 & $16 / 32$ & 16 & 64 & $>128$ & $>128$ \\
\hline$(R)-1$ & 16 & ND & 16 & 32 & $>128$ & $>128$ \\
\hline (S)-1 & 16 & ND & 16 & 64 & $>128$ & $>128$ \\
\hline$(R)-2$ & 16 & $8 / 16$ & 16 & 16 & $>128$ & $>128$ \\
\hline (S)-2 & 16 & $8 / 16$ & 16 & 32 & $>128$ & $>128$ \\
\hline$(R)-3$ & 4 & $4 / 8$ & 4 & $>128$ & $>128$ & $>128$ \\
\hline (S)-3 & 4 & $4 / 4$ & 4 & 16 & $>128$ & $>128$ \\
\hline$(R)-4$ & 1 & $2 / 4$ & 1 & $>128$ & $>128$ & $>128$ \\
\hline$(S)-4$ & 2 & $2 / 4$ & 2 & $>128$ & $>128$ & $>128$ \\
\hline$(R)-5$ & 1 & $2 / 2$ & 1 & $>128$ & $>128$ & $>128$ \\
\hline$(S)-5$ & 1 & $2 / 4$ & 1 & $>128$ & $>128$ & $>128$ \\
\hline$(R)-6$ & 16 & ND & 16 & $>128$ & $>128$ & $>128$ \\
\hline$(S)-6$ & $>128$ & ND & $>128$ & $>128$ & $>128$ & $>128$ \\
\hline$(R)-7$ & 4 & $4 / 8$ & 4 & $>128$ & $>128$ & $>128$ \\
\hline$(S)-7$ & 4 & $4 / 8$ & 4 & $>128$ & $>128$ & $>128$ \\
\hline$(R)-8$ & 8 & ND & 8 & 8 & $>128$ & $>128$ \\
\hline$(S)-8$ & 8 & ND & 8 & 16 & $>128$ & $>128$ \\
\hline
\end{tabular}

Abbreviations: MIC, minimum inhibitory concentration; MRSA, methicillin-resistant $S$. aureus; $\mathrm{ND}$, not determined.

${ }^{\mathrm{a}} \mathrm{MIC}_{50} / \mathrm{MIC}_{90}$.

After this first screening of in vitro antibacterial activity, only $(R)$-enantiomers from 3, 4 and $\mathbf{5}$ were retained for further investigation, as they displayed the better MICs on Gram-positive bacteria of all MQ derivatives tested. Time-kill studies were carried out for $S$. aureus CIP 103.429 and S. aureus strain SAAM 27 (resistant to methicillin, ciprofloxacin and levofloxacin). Compound ( $R$ ) -5 was the most efficient, with a $99.9 \%$ decrease of the inoculum as early as $2 \mathrm{~h}$ after inoculation when the concentration was superior or equal to the MIC. Contrary to what was observed with compound $(R)-4$, no regrowth at $24 \mathrm{~h}$ was witnessed for $(R)-\mathbf{5}$ at concentrations equal or superior to the MIC (for detailed results see Supplementary Figure S1 in Supplementary Information). Previously, time-kill assays showed that MQ was rapidly lytic for $S$. aureus. ${ }^{1}$ The same result was found here with MQ derivatives. However, the actual antibacterial mechanism of action of MQ remains poorly understood but DNA gyrase inhibition, a mechanism of action described for fluoroquinolone, was not implied. ${ }^{1}$ Along with the retained activity of our compounds on fluoroquinolone-resistant $S$. aureus strains, this further reinforce the hypothesis of a different mechanism of action for MQ derivatives.

As the tested MQ derivatives were not active against $P$. aeruginosa, we wanted to investigate whether this lack of activity could be linked to an efflux of the molecules from the bacterial cytoplasm through pumps such as MexAB-OprM. ${ }^{10} \mathrm{PA} \beta \mathrm{N}$, a known inhibitor of $P$. aeruginosa efflux pumps, was then added for MIC determinations on $P$. aeruginosa DSM 1117 and AM 85 (fluroroquinolone-resistant strain presenting a characterized efflux). All MICs remained above $128 \mu \mathrm{g} \mathrm{ml}^{-1}$ for MQ as well as all of the other compounds tested. These results rule out efflux as a mechanism explaining the lack of sensitivity of $P$. aeruginosa to 4 -aminoalcohol quinolines. As MQ has also been described as a possible inhibitor of efflux pumps in E. coli and $P$. aeruginosa, ${ }^{3}$ we also used $P$. aeruginosa AM 85 strain to assess whether MQ derivatives would bear the same inhibitory activity when used in conjunction with ciprofloxacin, a known substrate for $P$. aeruginosa efflux pumps. ${ }^{10}$ Although $\mathrm{PA} \beta \mathrm{N}$ induced a ready decrease in the MIC of ciprofloxacin (from 16 to $1 \mu \mathrm{g} \mathrm{ml}^{-1}$ ), neither MQ nor MQ derivatives at concentrations ranging from 25 to $100 \mu \mathrm{g} \mathrm{ml}^{-1}$ were able to trigger a similar decrease (for details see Supplementary Tables S1 and S2 in Supplementary Information). The discrepancy witnessed for MQ with previously published results in the efflux inhibition ${ }^{3}$ might be due to a methodological difference, as we only used phenotypical screening, or to a possible importance of the side chain in the inhibition process.

Finally, as MQ is mainly metabolized by the liver, the cytotoxicity of MQ and MQ derivatives was evaluated on hepatocellular carcinoma HepG2 cells with an MTT (3-(4,5-dimethylthiazol-2-yl)-2,5diphenyltetrazolium bromide) assay. $\mathrm{IC}_{50} \mathrm{~s}$, concentrations killing $50 \%$ of the cells, were calculated after a 24-h incubation with MQ or MQ derivatives. Compounds $(R)-\mathbf{3},(R)-4$ and $(R)-5 \quad \mathrm{IC}_{50}$ values were found to be of $19.4,16.6$ and $13.4 \mu \mathrm{g} \mathrm{ml}^{-1}$, respectively. MQ was also tested on this cell line and gave an $\mathrm{IC}_{50}$ value of $11 \mu \mathrm{g} \mathrm{ml}^{-1}$, below its MIC values. The safety index of MQ derivatives therefore ranged between 3- and 19-fold for (R)-3 and (R)-5, respectively, better on this model than the one from their parent molecule, which is currently used as an antimalarial.

In summary, the MQ derivatives tested in this paper were found to be mainly active on Gram-positive bacteria with a gain of 4- to 16fold compared with MQ. This activity did not appear to depend on the stereochemistry of the asymmetric carbon at the position 11 of the molecule but rather on the length of the side chain, with compound (R)-5 being the most effective and less toxic, making it the best candidate for further modifications and/or development. Gramnegative bacteria were less sensitive to MQ derivatives as these were found to be inactive against $P$. aeruginosa but still enabling a gain in activity of up to eightfold as compared with MQ for E. coli. Against the latter species, compounds with the shorter side chain length were the most effective, contrary to what was observed with Gram-positive bacteria, and $(R)$-enantiomers tended to have slightly lower MICs than their $(S)$ counterparts. This observation vouched for a potential implication of stereochemistry in the target of MQ derivative compounds in Gram-negative bacteria and/or in the transport of these molecules in the bacterial cytoplasm. Further investigations are needed to thoroughly elucidate the underlying mechanism of action of these compounds as well as that of MQ. Additional modifications on the side chain are also planned, which could lead to compounds with lower MICs and gain information on the implication of the second asymmetric carbon at the position 12 of the molecules in their antibacterial activity.

\section{ACKNOWLEDGEMENTS}

The authors thank François Helle for his technical assistance in cultivating HepG2 cells. Alexia Jonet was supported by a research grant funded by the Conseil Régional de Picardie and the Centre National de la Recherche Scientifique.

1 Kunin, C. M. \& Ellis, W. Y. Antimicrobial activities of mefloquine and a series of related compounds. Antimicrob. Agents Chem. 44, 848-852 (2000).

2 Bermudez, L. E. et al. Mefloquine is active in vitro and in vivo against Mycobacterium avium complex. Antimicrob. Agents Chem. 43, 1870-1874 (1999).

3 Vidal-Aroca, F., Meng, A., Minz, T., Page, M.G.P. \& Dreier, J. Use of resazurin to detect mefloquine as an efflux-pump inhibitor in Pseudomonas aeruginosa and Escherichia coli. J. Microbiol. Meth. 79, 232-237 (2009). 
4 Schlagenhauf, P., Adamcova, M., Regep, L., Schaerer, M.T. \& Rhein, H. G. The position of mefloquine as a 21st century malaria chemoprophylaxis. Malar. J. 9, 357 (2010).

5 Karle, J. M., Olmeda, R., Gerena, L. \& Milhous, W. K. Plasmodium falciparum: role of absolute stereochemistry in the antimalarial activity of synthetic amino alcohol antimalarial agents. Exp. Parasitol. 76, 345-351 (1993).

6 Mullié, C., Jonet, A., Desgrouas, C., Taudon, N. \& Sonnet, P. Differences in antimalarial activity of 4-aminoalcohol quinoline enantiomers and investigation of the presumed underlying mechanism of action. Malar. J. 11, 65 (2012).

7 Bermudez, L. E. et al. Identification of the $(+)$ - erythro-mefloquine as the active enantiomer with greater efficacy than mefloquine against Mycobacterium avium infection in mice. Antimicrob. Agents Chemother. 56, 4202-4206 (2012).

8 Shepherd, J. Use of $(+)$ mefloquine for the treatment of malaria. International Patent W098/39003 (1998).

9 Jonet, A. et al. First enantioselective synthesis of 4-aminoalcohol quinoline derivatives through a regioselective SN2 epoxide opening mechanism. Tetrahedron Asymmetry 22, 138-148 (2011)
10 Sonnet, P., Izard, D. \& Mullié, C Prevalence of efflux-mediated ciprofloxacin and levofloxacin resistance in recent clinical isolates of Pseudomonas aeruginosa and its reversal by the efflux pump inhibitors 1-(1-naphthylmethyl)-piperazine and phenylalanine-arginine- $\beta$-naphthylamide. Int. J. Antimicrob. Agents 39, 77-80 (2012).

11 Clinical and Laboratory Standards Institute Methods for Dilution Antimicrobial Susceptibility Tests for Bacteria that Grow Aerobically; approved standard. 8th edn Document M07-A8CLSI, Wayne, PA, USA (2009).

12 Griffin, C. E. et al. Mutation in Plasmodium falciparum CRT protein determines the stereospecific activity of antimalarial Cinchona alkaloids. Antimicrob. Agents Chemother. 56, 5356-5364 (2012).

13 Lipinski, C. A. Lead- and drug-like compounds: the rule-of-five revolution. Drug Discov. Today 1, 337-341 (2004).

14 Gutman, J. et al. Mefloquine pharmacokinetics and mefloquine-artesunate effectiveness in Peruvian patients with uncomplicated Plasmodium falciparum malaria. Malar. J. 8, 58 (2009).

Supplementary Information accompanies this paper on The Journal of Antibiotics website (http://www.nature.com/ja) 